\title{
Erectile problems in teenagers commonly miscategorized as psychogenic
}

Clinicians commonly assume that erectile dysfunction (ED) in younger males has psychological origins. A new study debunks this age-based bias, finding that a significant number of teenagers presenting with ED have underlying vascular abnormalities. "ED in teenagers should be evaluated with vascular studies before [being] assumed to be psychogenic," suggests lead author Raanan Tal, "as these studies may reveal the underlying etiology."

Hemodynamic assessment of older men with ED is routine, as the prevalence of common vascular risk factors, such as diabetes and hypertension, increases with age. By contrast, vascular impairmentmore likely to be related to trauma than to slowly accrued damage-is often excluded from the differential diagnosis of teenage ED.
Tal et al. retrospectively analyzed data from 40 males (mean age 18.1 years) who had presented to one of three specialist institutions in the US. The average lag time between onset of ED and presentation was nearly 2 years. The investigators found that 15 of the teenagers had a history of perineal trauma, and 6 had suffered penile injury or undergone penile surgery. Only 25 had been subject to vascular assessment in the form of cavernosography, dynamic infusion cavernosometry and/or penile duplex Doppler ultrasonography.

Vascular abnormalities - ten venous and two arterial-were detected. Five patients were deemed suitable for crural ligation surgery, and another two required further angiographic evaluation. Interestingly, only self-perceived erection quality, scored on a $0-100$ visual analog scale, differed significantly between those with and those without abnormal hemodynamic studies (40.6 versus 62.1, $P=0.036)$. No other aspect of patient history-including previous trauma or surgery, or presence of vascular risk factors-was predictive of underlying vascular pathology.

Incorrect diagnosis of psychogenic ED in teenagers is likely to have a detrimental effect on sexual confidence and self esteem. The authors suggest that performing appropriate vascular studies will optimize diagnostic accuracy, thereby improving treatment efficacy and prognosis.

Sarah Payton

Original article Tal, R. et al. Vasculogenic erectile dysfunction in teenagers: a 5 -year multi-institutional experience. BJU Int. 103, 646-650 (2009). 